\title{
Lasing of Coupled Guided Modes in Modified Hollow Hexagonal Semiconductor Cavities
}

\author{
Hee-Jong Moon ${ }^{1 *}$, Jin-Woong Lee ${ }^{1}$, Kyung-Sook Hyun ${ }^{1}$, and Dae Cheol Jeong ${ }^{2}$ \\ ${ }^{1}$ Department of Optical Engineering, Sejong University, Seoul 143-747, Korea \\ ${ }^{2}$ OE Solutions Co., Ltd., Gwangju 500-706, Korea
}

(Received April 14, 2014 : revised June 25, 2014 : accepted June 26, 2014)

\begin{abstract}
Coupled guided modes, proposed in various modified hollow hexagonal cavities each attached internally to a hexagon, were demonstrated by investigating the laser oscillations in semiconductor cavities. The mode spacing between two adjacent lasing peaks decreased as the size of the internal hexagon increased, due to the increased round-trip length of the coupled guided modes. The linear dependency of the inverse mode spacing to the calculated round-trip length strongly confirmed the lasing of the coupled guided modes. The proposed modes in common-sized external cavities showed resonance structure that could be adjusted widely by controlling the size of the internal hexagon.
\end{abstract}

Keywords : Coupled guided modes, Hollow hexagon, Microcavity laser

OCIS codes : (140.3948) Microcavity devices; (140.3410) Laser resonators

\section{INTRODUCTION}

Polygonal microcavities such as squares and hexagons, and polygonal ring cavities such as square ring cavities have attracted a great deal of interest due to the existence of whispering gallery (WG)-like modes, similar to WG modes in disk-type cavities, originating from the total internal reflection (TIR) of light at the boundaries [1-7]. Microcavities based on WG or WG-like modes are considered to be potential building blocks in photonic integrated circuits for optical filtering, switching, and microlasers [8-12]. Because the aforementioned cavities have flat sides, they present strong advantages, such as a long interaction length during coupling with external flat waveguides $[13,14]$.

Recently, a different type of WG-like modes called "guided modes" have been reported in hollow polygonal (square or hexagonal) geometries, wherein TIR occurs not only at the outer boundaries but also at the inner boundaries [15-17]. Figure 1 shows the previous hollow hexagonal cavity (outer size $d_{2}$, inner size $d_{1}$, and refractive index $m$ ) and the ray trajectory corresponding to the guided modes [17].

If the inner size $d_{1}$ is quite a bit smaller than $d_{2}$, WG-like modes are expected to bounce 6 times only at the outer boundaries, with an incident angle of $60^{\circ}$. If $d_{1}$ is slightly smaller than $d_{2}$, the possible modes are no longer the 6-bounce ones. Under the condition of $3 d_{2} / 4<d_{1}<6$ $d_{2} / 7,18$-bounce WG-like modes called "guided modes" are selected as the resonance modes, as shown in Fig. 1 [17]. The optical path length $L$ during one round trip, is calculated as [17]

$$
L=3 m\left(2 d_{2}-d_{1}\right) \equiv m l
$$

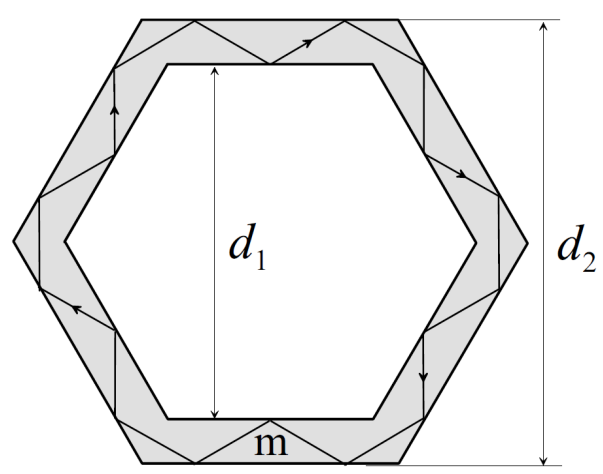

FIG. 1. Ray trajectory for 18-bounce guided modes in a hollow hexagonal cavity (outer size $d_{2}$, inner size $d_{1}$, and refractive index $m$ ) with an incident angle of $60^{\circ}$.

\footnotetext{
*Corresponding author: hjmoon@sejong.ac.kr

Color versions of one or more of the figures in this paper are available online.
} 
where $l$ is the round-trip length of the guided modes. The resonance wavelength $\lambda_{q}$ can be approximately determined from a ray-optics picture as

$$
\frac{2 \pi}{\lambda_{q}} L+18 \Psi\left(60^{\circ}\right) \cong q(2 \pi)
$$

where $q$ is an integer, and $\Psi\left(60^{\circ}\right)$ is the phase change during total internal reflection at the outer or inner boundaries. Equation (2) has a limitation in calculating the exact $\lambda_{q}$ because there is a large number of bounces during one round trip, where each bouncing produces the GoosHänchen effect [18]. The mode spacing $\Delta \lambda\left(=\lambda_{q-1}-\lambda_{q}\right)$ between two adjacent modes can be derived from Eq. (2).

One advantage of the guided modes is the tunable property of the resonance structure achieved by adjusting inner size $d_{1}$ for a common outer size $d_{2}$. Thus the roundtrip length $l$ or the mode spacing $\Delta \lambda$ can be also adjusted by changing the inner size $d_{1}$. Therefore, it is expected that a hollow polygonal cavity can be a tunable building block for photonic integrated circuits. By standardizing the outer size, multiple cavities with different mode structures can be simultaneously coupled to a straight waveguide or pairs of straight waveguides, which permits additional functionality for the resonance structure of the coupled cavities.

However, there is a limitation for the guided modes in a hollow polygonal cavity in an actual structure: The adjustable range $\delta \lambda$ in $\Delta \lambda$ is very small because the guided modes exist in a very narrow range of inner size $d_{1}$ for a fixed outer size $d_{2}$. In a rough calculation, the adjustable range $\delta \lambda$ in a hollow hexagonal cavity is limited to only $\sim 5 \%$ of $\Delta \lambda(\delta \lambda / \Delta \lambda \sim 0.05)$. Hence, the guided modes in a hollow polygonal cavity are limited to certain fine-tuning applications.

\section{PROPOSED COUPLED GUIDED MODES}

In this study, we propose a modified guided mode (denoted as a "coupled guided mode") with a very large adjustable range in a modified hollow polygonal cavity, wherein a polygon is attached internally, so that the guided modes extend to the entire cavity. An example is shown in Fig. 2, in which a hexagon B (size $d_{3}$ ) is attached at the center of an inner side of external hollow hexagonal cavity A (outer size $d_{2}$, inner size $d_{1}$ ).

The guided modes in Fig. 1 extend to internal hexagon $\mathrm{B}$, as depicted in Fig. 2. It is notable that there is no loss experienced by the light passing through the joint region, and this scheme is not limited in the wavelength of light. The elongation of the round-trip length by simply attaching the additional cavity is difficult to realize with other cavity geometries, such as MMI-coupled racetrack geometries [19]. If two racetrack cavities are coupled by MMI with a power coupling coefficient of $\kappa \cong 1$, light can jump from one cavity to the other at the MMI coupler,

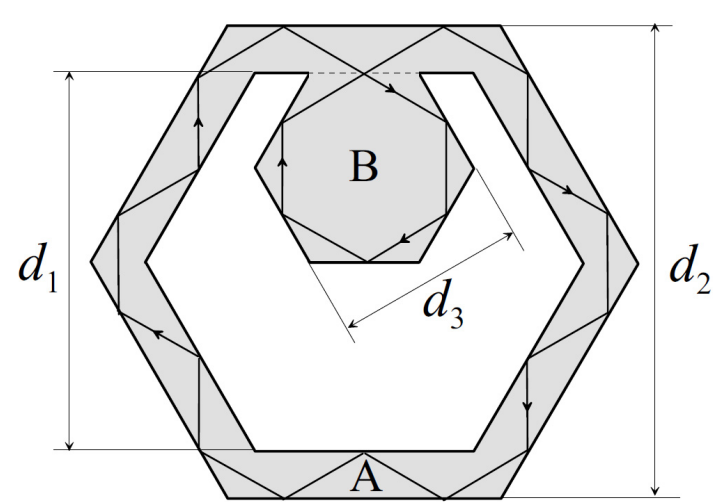

FIG. 2. Proposed modified hollow hexagonal external cavity, in which a hexagon (size $d_{3}$ ) is attached internally. The corresponding ray trajectory for 22-bounce coupled guided modes is also shown.

resulting in the extension of the cavity modes, but the wavelengths satisfying $\kappa \cong 1$ are limited to a small range because $\kappa$ depends on the wavelength of the light. Moreover, there is the insertion loss of the MMI coupler. Thus the proposed scheme cannot be directly applied to racetrack geometries. The optical path length $L$ corresponding to the resultant modes increases from Eq. (1) as

$$
L=3 m\left(2 d_{2}-d_{1}+d_{3}\right) \equiv m l
$$

Because $d_{3}$ can be varied over a wide range (from 0 to about $d_{1}$ ) for a given hollow hexagonal cavity A, a large change in the mode spacing $\Delta \lambda$ is expected for the coupled guided modes. The calculated adjustable range $\delta \lambda$ in $\Delta \lambda$ can amount to about $60 \%$ of $\Delta \lambda$, which is much larger than the aforementioned $5 \%$, just by controlling $d_{3}$ for a given external cavity. Therefore, the proposed cavity could be a versatile building block with large scalability in the resonance structure. In applications for switching, fine tuning of resonance modes might be also required by locally changing the refractive index of the cavity via current or heat. The modified cavity in Fig. 2 is ideal for this application because the fine-tuning functionality can be realized specifically on the internal hexagon B. In this study, we demonstrated the lasing of the proposed coupled guided modes in a modified hollow hexagonal semiconductor cavity and investigated the dependence of the lasing spectrum on the internal size.

\section{EXPERIMENTS}

The experimental setup and epistructure used for the semiconductor cavities were similar to those in our previous studies [16, 17]. InGaAsP/InGaAs multiple quantum wells (MQWs, $\lambda_{\mathrm{g}} \cong 1.55 \mu \mathrm{m}$ ) surrounded by two confinement layers were used for the laser gain medium. The total thickness of the epistructure formed on an InP substrate 
was about $3 \mu \mathrm{m}$. Various cavities were lithographically patterned and fabricated by inductively coupled plasma (ICP) etching, unlike the previous RIE (reactive ion etching) process. The total etching depth of about $4 \mu \mathrm{m}$ was sufficient for lasing. A pulsed $\mathrm{Nd} \mathrm{YVO}_{4}$ laser (wavelength $1.06 \mu \mathrm{m}$, repetition rate $10 \mathrm{kHz}$, pulse width $300 \mathrm{~ns}$ ) was loosely focused on a cavity to optically pump the MQWs. The illuminated cavity was monitored using a CCD camera attached to a high-resolution microscope. A tapered fiber approached a corner of the illuminated cavity to collect the lasing signal; the other end of the tapered fiber was connected to an optical spectrum analyzer for measurement of the lasing spectrum. We confirmed that the measured signal is due to lasing by observing the threshold behavior of the spectral peak intensity. The threshold laser pulse energy was about $2 \mu \mathrm{J}$ when the illuminated spot size of the pulsed pump beam on the wafer was about $500 \mu \mathrm{m}$, which corresponded to a peak pump intensity of $\sim 3 \mathrm{~kW} / \mathrm{cm}^{2}$.

\section{RESULTS \& DISCUSSIONS}

Figure 3 shows typical lasing spectra, measured at $\sim 3$ times the threshold pump level, for hollow hexagonal external cavities $\left(d_{2}=80 \mu \mathrm{m}, d_{1}=64 \mu \mathrm{m}\right)$ with various sizes $d_{3}$ of the internal hexagon. It is notable that parameters $d_{2}$ and $d_{1}$ satisfy the condition for guided modes in Fig. 1. For the case of $d_{3}=0$ (no internal hexagon), the lasing peaks appeared around $1570 \mathrm{~nm}$ with a mode spacing $\Delta \lambda$ of about $2.42 \mathrm{~nm}$, as shown in Fig. 3(a). The lasing modes should be the 18-bounce guided modes of Fig. 1. For the cases of $d_{3}=30 \mu \mathrm{m}$ and $50 \mu \mathrm{m}$ the mode spacing was reduced to about $1.83 \mathrm{~nm}$ and 1.56 $\mathrm{nm}$ respectively, as shown in Fig. 3. (b) and (c). The reduction of the mode spacing implied that the optical path length increases with the size of the internal hexagon. Therefore, the lasing modes should not be the 18-bounce guided modes of Fig. 1 but could be the 22-bounce coupled guided modes shown in Fig. 2.

The mode spacing $\Delta \lambda$ of the resonance modes in a semiconductor laser can be expressed as $[16,17]$

$$
\Delta \lambda \cong \frac{\lambda^{2}}{m\left[1-\left(\frac{\lambda}{m}\right)(d m / d \lambda)\right] l} \equiv \frac{\lambda^{2}}{M(\lambda) l}
$$

where the group index $M(\lambda)$ accounts for the dispersion in the semiconductor material and is weakly dependent on $\lambda$.

Because all the center wavelengths of the lasing spectra were similarly observed around $1565 \mathrm{~nm}$ in Fig. 3, the group index $M(\lambda)$ can be considered constant. Thus the mode spacing in Eq. (4) should be inversely proportional to the round-trip length $l$ of the resonance modes. If the lasing peaks in Fig. 3(b) are the proposed coupled guided modes, the mode-spacing ratio $(2.42 \mathrm{~nm} / 1.83 \mathrm{~nm} \cong 1.32)$ between Fig. 3(a) and (b) should be equal to the inverse ratio of the corresponding l's. The calculated inverse ratio of $l(378 \mu \mathrm{m} / 288 \mu \mathrm{m} \cong 1.31)$, using Eq. (4) and (3), is essentially the same as the measured mode-spacing ratio of

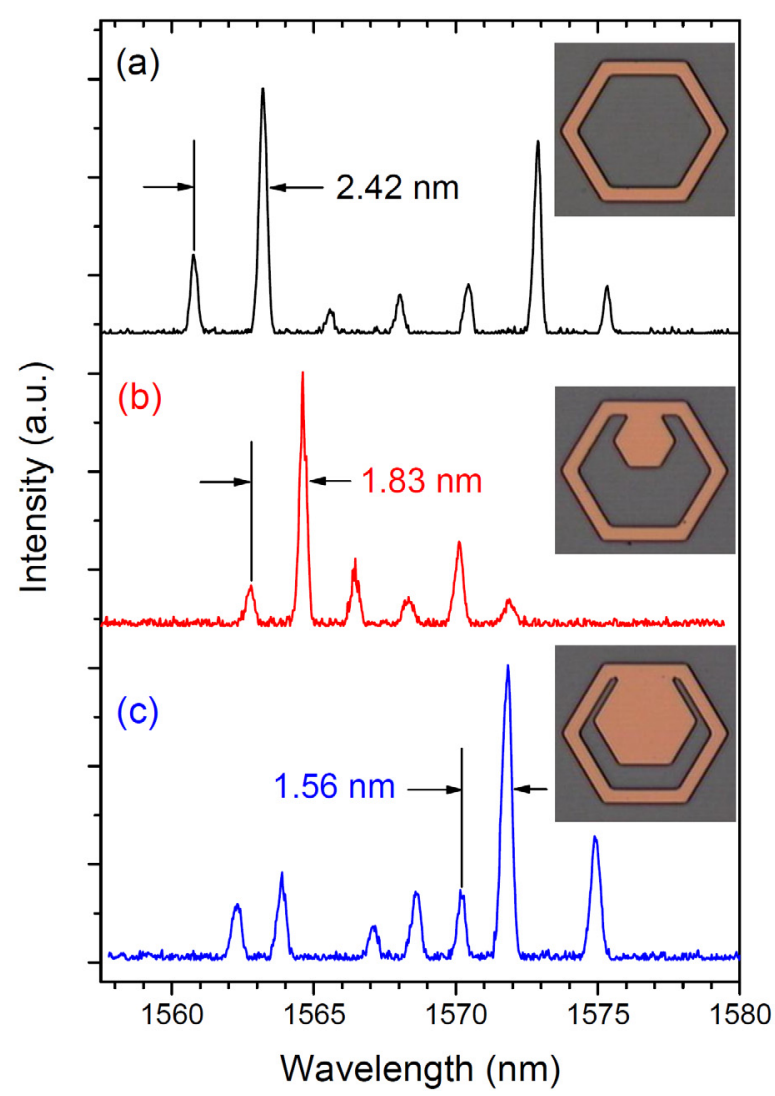

FIG. 3. Typical lasing spectra of modified hollow hexagonal semiconductor cavities $\left(d_{2}=80 \mu \mathrm{m}, d_{1}=64 \mu \mathrm{m}\right)$ with internal hexagons of (a) $d_{3}=0$, (b) $d_{3}=30 \mu \mathrm{m}$, (c) $d_{3}=50 \mu \mathrm{m}$. Microscopic images of the cavities are also shown.

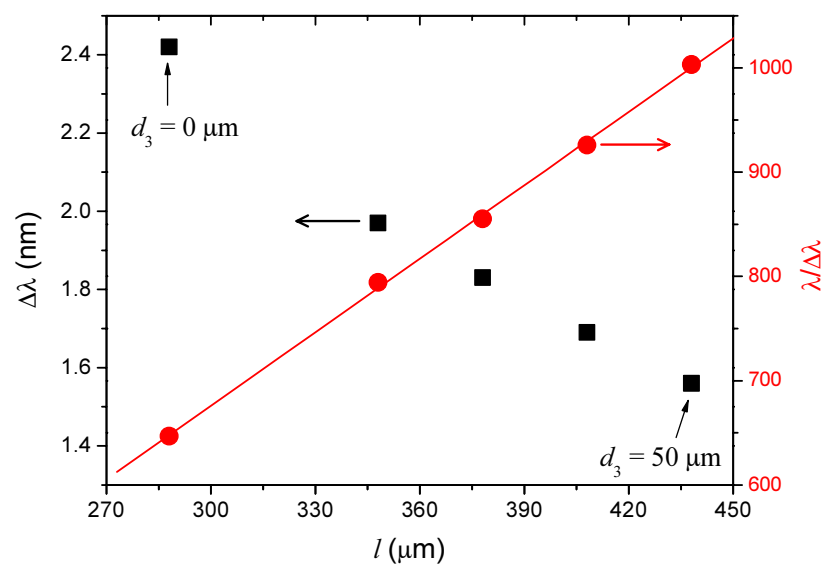

FIG. 4. Dependence of measured mode spacing $\Delta \lambda$ (black squares) and $\lambda / \Delta \lambda$ (red circles) on the calculated round-trip length $l$ for common external cavities $\left(d_{2}=80 \mu \mathrm{m}, d_{1}=64\right.$ $\mu \mathrm{m})$ with various $d_{3}(0,20,30,40,50 \mu \mathrm{m})$. 
1.32. Therefore, the lasing modes should be the coupled guided modes as conjectured.

Figure 4 summarizes the mode spacing $\Delta \lambda$ measured at $\lambda \sim 1565 \mathrm{~nm}$ from the common external cavities $\left(d_{2}=80\right.$ $\left.\mu \mathrm{m}, d_{1}=64 \mu \mathrm{m}\right)$ with different $d_{3}(0,20,30,40$, and 50 $\mu \mathrm{m})$. In Fig. 4, the dependence of $\lambda / \Delta \lambda$ on the round-trip length $l$ in Eq. (3) is also shown. $\lambda / \Delta \lambda$ showed a linear dependence on $l$ with a slope of $2.349\left(\mu \mathrm{m}^{-1}\right)$. Therefore, $M(\lambda)$ in Eq. (4) was derived as $2.349\left(\mu \mathrm{m}^{-1}\right) 1.565 \mu \mathrm{m} \cong$ 3.67. This is slightly larger than the previously reported value, due to the different epistructure and center wavelengths of the lasing [16]. The linear relation between $\lambda / \Delta \lambda$ and $l$ in Fig. 4 strongly confirms the lasing of the coupled guided modes in Fig. 2.

In Fig. 4 , the mode spacing $\Delta \lambda$ gradually decreased as $d_{3}$ increased. It is notable that $\Delta \lambda$ varied over a wide range, i.e. the adjustable range $\delta \lambda$ was very large. For example, $\Delta \lambda$ changed about $55 \%$ from the case of $d_{3}=0$ to that of $d_{3}=50 \mu \mathrm{m}$ (i.e. $2.42 \mathrm{~nm} / 1.56 \mathrm{~nm} \sim 1.55$ ). Therefore, the coupled guided modes showed a widely adjustable resonance structure, as proposed.

To verify whether coupled guided modes exist for other sizes of the external cavity, various other external cavities were also designed and fabricated. Figure 5 shows typical lasing spectra with different common sizes $\left(d_{2}=100 \mu \mathrm{m}\right.$, $\left.d_{1}=80 \mu \mathrm{m}\right)$. As $d_{3}$ of the internal hexagon increases from 0 to $20 \mu \mathrm{m}$ to $60 \mu \mathrm{m}$, the mode spacing $\Delta \lambda$ of the lasing peaks decreased from about $1.95 \mathrm{~nm}$ (Fig. 5(a)) to $1.7 \mathrm{~nm}$ (Fig. 5(b)) and $1.26 \mathrm{~nm}$ (Fig. 5(c)) respectively. The reduction of the mode spacing should be also interpreted as the lasing of the coupled guided modes in Fig. 2, as observed in Fig. 3.

The 18-bounce guided mode lasing in Fig. 5(a) appeared at $\sim 1573 \mathrm{~nm}$. The lasing peaks in Fig. 5(a) seem to be irregular as the mode spacing between several peaks on the left was not the same. This irregular feature should be attributed to the simultaneous lasing of coupled guided modes with two different polarizations (TE and TM). It is notable that the center wavelength of the lasing in Fig. 5(b) appeared at $\sim 1563 \mathrm{~nm}$, which is quite a bit shorter than that in Fig. 5(a). The center wavelength tends to appear in a lower absorption region (longer wavelength) as the total cavity loss decreases, as explained previously [17]. Thus the blue-shift of the center wavelength implies that the total cavity loss of the resonance modes increased. One reason might be the larger bouncing number of the 22-bounce coupled guided modes than the 18-bounce guided modes. More frequent bouncing during one round trip will yield a much greater total loss because scattering loss in the TIR process comes from the roughness of the etched surface. However, this seems unlikely because an apparent blue-shift was not observed in Fig. 3 for the same increase in bouncing number.

The other plausible origin is due to the smaller side length of the internal hexagon. Due to the Goos-Hänchen

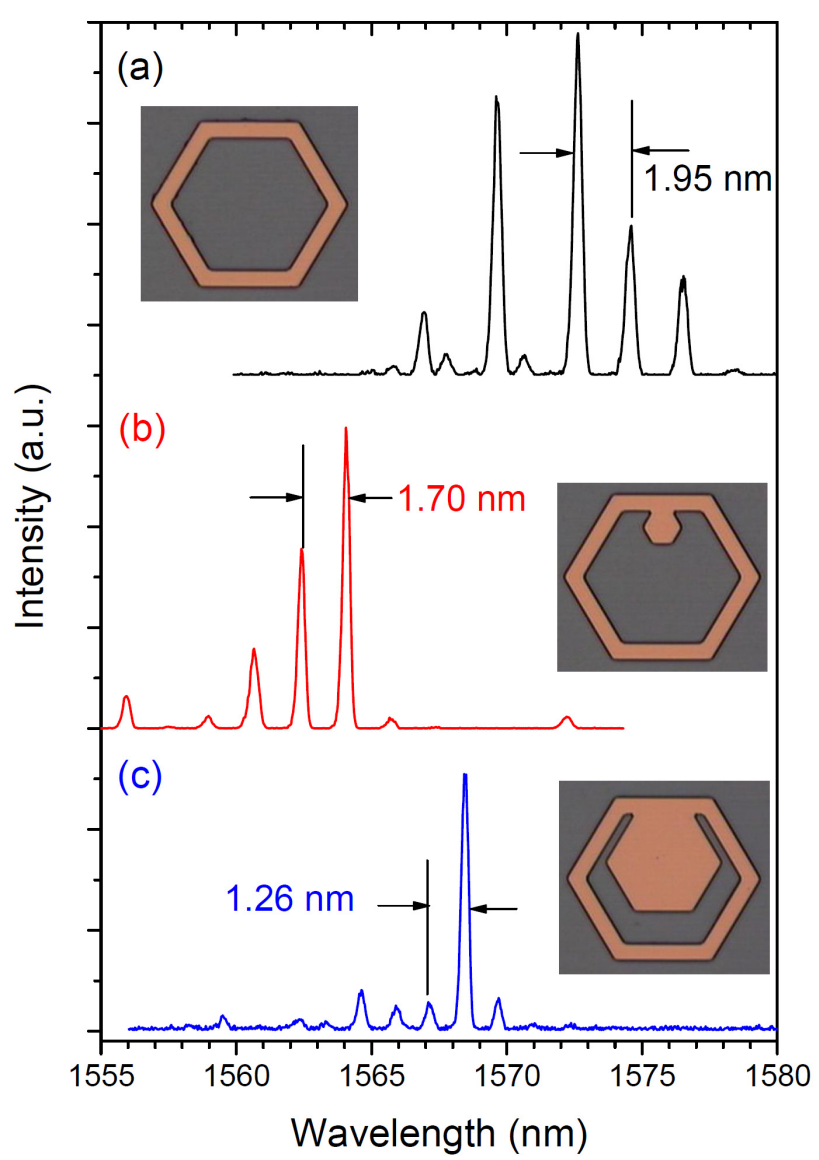

FIG. 5. Typical lasing spectra of modified hollow hexagonal semiconductor cavities $\left(d_{2}=100 \mu \mathrm{m}, d_{1}=80 \mu \mathrm{m}\right)$ with internal hexagons of (a) $d_{3}=0$, (b) $d_{3}=20 \mu \mathrm{m}$, (c) $d_{3}=60 \mu \mathrm{m}$.

effect, a ray-propagation distance exists for the TIR process at the flat boundary [18]. Thus an evanescent wave exists along the flat side within a finite length of propagation. The evanescent wave could leak from the boundary corner. As the flat side length of the internal hexagon becomes smaller, more evanescent leakage loss from the corner is expected. Therefore, the quality factor $\mathrm{Q}$ of the coupled guided modes will be rapidly spoiled when $d_{3}$ becomes smaller than a certain value. The Q-spoiling in Fig. 5(b) can be attributed dominantly to this evanescent leakage loss. Thus too small $d_{3}$ should be avoided for high-Q coupled guided modes in real applications.

\section{CONCLUSION}

In conclusion, resonance modes called coupled guided modes were proposed in a hollow polygonal cavity in which an additional polygon is attached internally. In the case of a hollow hexagonal cavity, 18-bounce guided modes were modified to 22-bounce coupled guided modes using an internal hexagon to extend the round-trip length. The proposed coupled guided modes were demonstrated by 
investigating the lasing spectra in various semiconductor cavities. The adjustable range in the mode spacing became about 10 times larger than for the previous guided modes, which will be beneficial for tunable photonic building blocks with common outer size. This scheme can be expanded to different polygonal shapes, such as square and octagonal geometries.

\section{ACKNOWLEDGMENT}

This research was supported by Basic Science Research Program through the National Research Foundation of Korea (NRF) funded by the Ministry of Education, Science and Technology (2010-0022331)

\section{REFERENCES}

1. A. W. Poon, F. Courvoisier, and R. K. Chang, "Multimode resonances in square-shaped optical microcavities," Opt. Lett. 26, 632-634 (2001).

2. Y. Z. Huang, K. J. Che, Y. D. Yang, S. J. Wang, Y. Du, and Z. C. Fan, "Directional emission InP/GaInAsP squareresonator microlasers," Opt. Lett. 33, 2170-2172 (2008).

3. N. Ma, C. Li, and A. W. Poon, "Laterally coupled hexagonal micropillar resonator add-drop filters in silicon nitride," IEEE Photon. Technol. Lett. 16, $2487-2489$ (2004).

4. D. J. Gargas, M. C. Moore, A. Ni, S. W. Chang, Z. Zhang, S. L. Chuang, and P. Yang, "Whispering gallery mode lasing from zinc oxide hexagonal nanodisks," ACS Nano 4, 3270-3276 (2010).

5. K. S. Hyun and H. J. Moon, "Resonant characteristics of multimode interferometer coupled square ring semiconductor resonators," Opt. Express 18, 6382-6389 (2010).

6. K. S. Hyun, T. Lee, and H. J. Moon, "Single mode lasing in InGaAsP/InP semiconductor coupled square ring cavities," J. Opt. Soc. Korea 16, 157-161 (2012).
7. D. K. Armani, T. J. Kippenberg, S. M. Spillane, and K. J. Vahala, "Ultra-high-Q toroid microcavity on a chip," Nature 421, 925-928 (2003).

8. A. A. Savchenkov, V. S. Ilchenko, A. B. Matsko, and L. Maleki, "High-order tunable filters based on a chain of coupled crystalline whispering gallery-mode resonators," IEEE Photon. Technol. Lett. 17, 136-138 (2005).

9. M. Cai, O. Painter, and K. J. Vahala, "Observation of critical coupling in a fiber taper to a silica-microsphere whispering-gallery mode system,” Phys. Rev. Lett. 85, 74-77 (2000).

10. V. Van, T. A. Ibrahim, K. Ritter, P. P. Absil, F. G. Johnson, R. Grover, J. Goldhar, and P.-T. Ho, "All-optical nonlinear switching in GaAs-AlGaAs microring resonators," IEEE Photon. Technol. Lett. 14, 74-76 (2002).

11. H. J. Moon, Y. T. Chough, and K. An, "Cylindrical microcavity laser based on the evanescent-wave-coupled gain,” Phys. Rev. Lett. 85, 3161-3164 (2000).

12. S. M. Spillane, T. J. Kippenberg, and K. J. Vahala, "Ultralow-threshold Raman laser using a spherical dielectric microcavity," Nature 415, 621-623 (2002).

13. H. J. Moon and K. S. Hyun, "Bow-tie mode lasing in a grooved rectangular semiconductor microcavity," J. Opt. Soc. Korea 16, 162-165 (2012).

14. C. Y. Fong and A. W. Poon, "Planar corner-cut square microcavities: Ray optics and FDTD analysis," Opt. Express 12, 4864-4874 (2004).

15. H. J. Moon, S. P. Sun, J. H. Lee, and K. An, "Guided mode lasing in a double-layered square microcavity," Appl. Phys. Lett. 83, 2521-2523 (2003).

16. H. J. Moon and K. S. Hyun, "Selective lasing of guided modes from hollow square semiconductor microcavties," Jpn. J. Appl. Phys. 46, L274-L276 (2007).

17. H. J. Moon, "Enhanced lasing characteristics of guided modes in an epistructured hollow hexagonal cavity," Jpn. J. Appl. Phys. 48, 030205 (2009).

18. B. E. A. Saleh and M. C. Teich, Fundamentals of Photonics (John Wiley, 1991), Chapter 7.

19. D. G. Rabus and M. Hamacher, "MMI-coupled ring resonators in GaInAsP-InP," IEEE Photon. Technol. Lett. 13, 812-814 (2001). 